\title{
Serum Chlorine Level as a Possible Predictive Factor for Oxaliplatin-Induced Peripheral Neuropathy*
}

\author{
Satoshi Tanaka ${ }^{1,2}$, Naoto Suzuki ${ }^{1}$, Akira Mimura ${ }^{1}$, Maho Kurosawa ${ }^{1}$, Yuriko Murai ${ }^{3}$, Daisuke Saigusa ${ }^{1}$, \\ Makio Gamoh ${ }^{4}$, Masuo Sato ${ }^{2}$, Yoshihisa Tomioka ${ }^{1,5}$ \\ ${ }^{1}$ Laboratory of Oncology, Pharmacy Practice and Sciences, Graduate School of Pharmaceutical Sciences, Tohoku University, Sendai, \\ Japan; ${ }^{2}$ Department of Pharmacy, South Miyagi Medical Center, Ohgawara, Japan; ${ }^{3}$ Pharmacy Education and Research Center, \\ Graduate School of Pharmaceutical Sciences, Tohoku University, Sendai, Japan; ${ }^{4}$ Department of Medical Oncology, South Miyagi \\ Medical Center, Ohgawara, Japan; ${ }^{5}$ Department of Community Pharmacy, Graduate School of Pharmaceutical Sciences, Tohoku \\ University, Sendai, Japan. \\ Email: ytomioka@m.tohoku.ac.jp
}

Received September $24^{\text {th }}, 2011$; revised November $27^{\text {th }}, 2011$; accepted December $9^{\text {th }}, 2011$

\begin{abstract}
Peripheral neuropathy is a major adverse event associated with oxaliplatin-based chemotherapy and is a major dose-limiting adverse event in clinical practice. However, some patients treated with oxaliplatin may show no or minimal peripheral neuropathy. These differences are still poorly understood. The data on patients with colorectal cancer who received oxaliplatin-based regimens between January 2005 and June 2010 at South Miyagi Medical Center were retrospectively retrieved from the medical records. We selected 51 patients, and factor analysis was performed. The serum chlorine $(\mathrm{Cl})$ level at baseline was significantly higher in patients with a high frequency of peripheral neuropathy (106; range $104-107$ vs. 104; range $101-104 \mathrm{mEq} / \mathrm{L}, \mathrm{p}=0.02$ ). Principal component analysis showed the variables $\mathrm{Cl}$, body mass index, status of liver metastasis, and status of lymph node metastasis were related to the incidence of peripheral neuropathy. Discriminant analysis showed the model had predicted $72.5 \%$ of peripheral neuropathy. An understanding of the patient's characteristics could be useful for preventing or predicting oxaliplatin-induced peripheral neuropathy.
\end{abstract}

Keywords: Peripheral Neuropathy; Oxaliplatin; Chlorine; Predicting Factors; Predictive Model

\section{Introduction}

Currently, colorectal cancer treatment is performed by surgery/endoscope treatment and chemotherapy. After chemotherapy with fluorouracil (5-FU) was first developed in 1957, 5-FU with a folic acid/leucovorin (LV) regimen based on a concept of biochemical modulation was used as standard therapy. Prolonged survival was achieved using combination therapies with folic acid, 5-FU and oxaliplatin (FOLFOX) or with folic acid, 5-FU and irinotecan (FOLFIRI) regimens. The efficacy against colorectal cancer has been enhanced by the development of molecular-targeted drugs such as bevacizumab, cetuximab and panitumumab [1-5].

Oxaliplatin [6], a third-generation platinum agent, is a key drug in the chemotherapy treatment of colorectal cancer. Many clinical trials with FOLFOX and XELOX (in combination with capecitabine and oxaliplatin) regimens have been shown to be useful. The National Comprehensive Cancer Network (NCCN) guidelines currently

*Conflict of Interest: None declared. recommend FOLFOX and XELOX \pm bevacizumab regimens as first-line therapy for colorectal cancer (http:// www.ncen.org).

According to the Food and Drug Administration (FDA) report and numerous clinical trials [1-5,7], however, oxaliplatin frequently induces characteristic patterns of peripheral neurotoxicity in the form of two distinct types [8]. The transient acute and cold-induced peripheral neuropathy experienced during administration or immediately after the administration of oxaliplatin is always reversible without persistent impairment of sensory nerve function, and this effect does not require the discontinuation of chemotherapy. However, when treatment is continued, the extent of the symptoms can increase, and their duration can be prolonged to the extent of becoming permanent. Chronic peripheral neuropathy, which is characterized by the loss of sensory and motor dysfunction after long-term treatment with oxaliplatin, is a major dose-limiting symptom. Although various neuromodulatory agents such as calcium-magnesium infusions $[9,10]$, $\alpha$-lipoic acid [11], venlafaxine [12,13], amifostine [14], 
glutathione [15], glutamine [16], carbamazepine [17], gabapentin [18] and pregabalin have been proposed to prevent or treat oxaliplatin-induced peripheral neuropathy, randomized trials demonstrating a therapeutic effect on oxaliplatin-induced peripheral nephropathy are lacking; additionally, the effectiveness of these agents is still obscure $[19,20]$. As indicated in the OPTIMOX study, the stop-and-go concept with intermittent administration of oxaliplatin to reverse oxaliplatin-induced neurotoxicity could be considered of value [21]. The predictability of oxaliplatin-induced peripheral neuropathy should not only allow medical staff to manage this adverse event but also improve the quality of life. Therefore, we investigated the predictive factors associated with the incidence and frequency of oxaliplatin-induced peripheral neuropathy.

\section{Materials and Methods}

\subsection{Patients}

This study was carried out as a retrospective analysis of 77 patients who had been treated with FOLFOX4 [2,22], mFOLFOX6 [23] or mFOLFOX6 plus bevacizumab at the South Miyagi Medical Center between January 2005 and June 2010. The clinical characteristics at baseline and any adverse events were determined from the electronic medical records or paper medical records. Adverse events (peripheral neuropathy, stomatitis, fatigue, anorexia, nausea, vomiting, diarrhea and constipation) were evaluated and recorded according to the guidelines of the National Cancer Institute Common Terminology Criteria for Adverse Events version 3.0 (NCI-CTCAE). Patients who received less than 4 courses of treatment, patients with missing data, or patients who were scheduled for the "Stop and Go strategy" therapy were excluded. The final study included 51 patients. Patients were categorized into two groups according to the frequency of peripheral neuropathy: the high-frequency group (high PN) or the lowfrequency group (low PN). In this study, the frequency of peripheral neuropathy was defined as the value of the total expression of peripheral neuropathy during treatment divided by the total number of treatment courses. The median of the frequency of peripheral neuropathy was $0.40(\mathrm{~N}=51)$. Patients were equally divided by this median (0.40) into the high PN (median 0.60, $0.45-0.71$, $\mathrm{N}=27$ ) and the low PN (median 0.19, $0.03-0.25, \mathrm{~N}=$ $24)$ groups. The study design was reviewed by a local ethics committee for research involving human subjects at the Graduate School of Pharmaceutical Sciences, Tohoku University and South Miyagi Medical Center.

\subsection{Data Analysis}

Information on gender; age; height; weight; body mass index (BMI); body surface area (BSA); type of cancer (rectum/colon/other); metastases (lung/liver/lymph node); surgical history; cancer chemotherapy history; radiotherapy history; chemotherapy regimen (mFOLFOX6/ mFOLFOX6 + bebacizmab/FOLFOX4); oxaliplatin dose per course; number of cycles of chemotherapy that the patient received; laboratory tests (total bilirubin (T.Bil), aspartate aminotransferase (AST), alanine aminotransferase (ALT), lactate dehydrogenase (LDH), alkaline phosphatase (ALP), $\gamma$-glutamyl transpeptidase $(\gamma$-GTP), blood urea nitrogen (BUN), serum creatinine ( $\mathrm{SCr}$ ), $\mathrm{Na}$, $\mathrm{K}, \mathrm{Cl}$ ); and use of medication (antihypertensive, reninangiotensin system inhibitor, $\mathrm{Ca}$ antagonist, narcoleptic, anti-microbial, anti-diabetic, anti-hyperlipidemic, laxative, prokinetic, nonsteroidal anti-inflammatory drugs (NSAIDs), anti-diarrheal, anti-peptic ulcer, anti-psychotropic, hematinic, narcotic, and anti-flatulent agent) was obtained from the medical records. Variables were compared using the $\chi^{2}$-test, Fisher's exact test and the Wilcoxon rank-sum test. For multivariate analysis, a princepal component analysis (PCA) and discriminant analysis were applied, and predictive model equations were estimated. The level of statistical significance was set at $\mathrm{P}<$ 0.05 . All statistical analyses were performed using PASW software version 17 (SPSS Japan Inc., Tokyo, Japan).

\section{Results}

\subsection{Frequency Distribution of Adverse Events}

The frequency distribution of adverse events is shown in Table 1. The maximum grade in all courses is presented as the grade for each patient. The incidence of adverse events was $88.2 \%$ for peripheral neuropathy, $49.0 \%$ for anorexia and $41.2 \%$ for nausea.

Table 1. Frequency distribution of adverse events $(\mathrm{N}=51)$.

\begin{tabular}{ccccc}
\hline \multirow{2}{*}{$\begin{array}{c}\text { Adverse } \\
\text { events }\end{array}$} & $\mathbf{5}$ & $\mathbf{1}$ & $\mathbf{2}$ & $\mathbf{3}$ \\
\cline { 2 - 4 } GNading $^{\mathrm{a}}$ \\
\hline Ptomatitis & $43(84.3)$ & $7(13.7)$ & $0(0)$ & $1(2.0)$ \\
Fatigue & $37(72.5)$ & $6(11.8)$ & $4(7.8)$ & $4(7.8)$ \\
Anorexia & $26(51.0)$ & $12(23.5)$ & $10(19.6)$ & $3(5.9)$ \\
Nausea & $30(58.8)$ & $10(19.6)$ & $6(11.8)$ & $5(9.8)$ \\
Vomit & $36(70.6)$ & $10(19.6)$ & $2(3.9)$ & $3(5.9)$ \\
Diarrhea & $39(76.5)$ & $9(17.6)$ & $2(3.9)$ & $1(2.0)$ \\
Constipation & $38(74.5)$ & $12(23.5)$ & $1(2.0)$ & $0(0)$ \\
\hline
\end{tabular}

${ }^{\mathrm{a}}$ Data presented as n (\%), ${ }^{\mathrm{b}}$ peripheral neuropathy. 


\subsection{Peripheral Neuropathy in the High-Frequency Group (High PN) and the Low-Frequency Group (Low PN)}

Table 2 shows the patient characteristics after patients were categorized to a high-frequency group of peripheral neuropathy $(\mathrm{N}=27)$ and a low-frequency group of peripheral neuropathy $(\mathrm{N}=24)$. Sex, age, BSA, type of cancer, metastatic status, past treatment history, type of regimen and the dose of oxaliplatin were not signifycantly different. The number of cycles of chemotherapy was significantly different $(\mathrm{P}=0.002)$.

\subsection{Laboratory Tests and Medications}

Table 3 shows the patient characteristics with respect to their laboratory tests at baseline. The serum chlorine $(\mathrm{Cl})$ level of the high-PN group was approximately $2 \mathrm{mEq} / \mathrm{L}$ higher than that of the low PN group $(\mathrm{P}=0.02)$. Other electrolytes and laboratory data were not significantly different. Table 4 shows the use of medication at baseline, and there was no significant difference between the low $\mathrm{PN}$ and the high PN groups.

Table 2. Patient characteristics at baseline.

\begin{tabular}{|c|c|c|c|}
\hline Characteristics & $\begin{array}{l}\text { Low PN } \\
(N=24)\end{array}$ & $\begin{array}{l}\text { High PN } \\
(\mathbf{N}=27)\end{array}$ & P value \\
\hline Male (\%) & 62.5 & 70.4 & 0.56 \\
\hline Age (years) ${ }^{\mathrm{a}}$ & $59(55-67)$ & $64(57-70)$ & 0.16 \\
\hline Height $(\mathrm{cm})^{\mathrm{a}}$ & $\begin{array}{c}160.8 \\
(155.2-168.1)\end{array}$ & $\begin{array}{c}162.5 \\
(157.0-166.7)\end{array}$ & 0.82 \\
\hline Weight $(\mathrm{kg})^{\mathrm{a}}$ & $\begin{array}{c}62.9 \\
(55.6-66.4)\end{array}$ & $\begin{array}{c}58.3 \\
(49.1-66.0)\end{array}$ & 0.16 \\
\hline $\operatorname{BMI}\left(\mathrm{kg} / \mathrm{m}^{2}\right)^{\mathrm{a}}$ & $\begin{array}{c}23.9 \\
(21.7-26.6)\end{array}$ & $\begin{array}{c}22.6 \\
(19.7-24.4)\end{array}$ & 0.08 \\
\hline $\operatorname{BSA}\left(\mathrm{m}^{2}\right)^{\mathrm{a}}$ & $\begin{array}{c}1.61 \\
(1.52-1.69)\end{array}$ & $\begin{array}{c}1.57 \\
(1.39-1.69)\end{array}$ & 0.29 \\
\hline Rectum/Colon/Other (\%) & $54.2 / 37.5 / 8.3$ & $55.6 / 40.7 / 3.7$ & 0.78 \\
\hline Metastasis $(\%)$ & 75.0 & 85.2 & 0.29 \\
\hline Lung metastasis (\%) & 29.2 & 18.5 & 0.37 \\
\hline Liver metastasis $(\%)$ & 45.8 & 66.7 & 0.13 \\
\hline Lymph node metastasis (\%) & 4.2 & 22.2 & 0.07 \\
\hline Surgical history (\%) & 91.7 & 92.6 & 1.0 \\
\hline Chemotherapy history (\%) & 25.0 & 14.8 & 0.49 \\
\hline Radiotherapy history (\%) & 4.2 & 0 & 0.47 \\
\hline Regimen $(1 / 2 / 3)^{\mathrm{b}}(\%)$ & $79.2 / 12.5 / 8.3$ & $77.8 / 14.8 / 7.4$ & 0.97 \\
\hline $\begin{array}{l}\text { Oxaliplatin dose per course } \\
\qquad(\mathrm{mg})^{\mathrm{a}}\end{array}$ & $\begin{array}{c}130 \\
(121-140)\end{array}$ & $\begin{array}{c}130 \\
(110-140)\end{array}$ & 0.51 \\
\hline Cycles patient received ${ }^{\mathrm{a}}$ & $7(5-9)$ & $10(8-12)$ & 0.002 \\
\hline
\end{tabular}

${ }^{\mathrm{a}}$ Data presented as median (range); ${ }^{\mathrm{b}}$ Regimen1, mFOLFOX6; regimen2, mFOLFOX6 + bebacizmab; regimen3, FOLFOX4.
Table 3. Patient characteristics for laboratory tests at baseline ${ }^{\mathrm{a}}$.

\begin{tabular}{cccc}
\hline Characteristics & $\begin{array}{c}\text { Low PN } \\
\mathbf{( N = 2 4 )}\end{array}$ & $\begin{array}{c}\text { High PN } \\
\mathbf{( N = 2 7 )}\end{array}$ & P value \\
\hline T.bil. $(\mathrm{mg} / \mathrm{dL})$ & $0.58(0.58-0.75)$ & $0.55(0.41-0.70)$ & 0.61 \\
AST (U/L) & $21(17-36)$ & $24(17-29)$ & 0.95 \\
ALT (U/L) & $21(14-40)$ & $20(13-30)$ & 0.62 \\
LDH (U/L) & $198(157-284)$ & $175(151-201)$ & 0.14 \\
ALP (U/L) & $317(222-471)$ & $287(184-423)$ & 0.42 \\
$\gamma$-GTP (U/L) & $38(23-135)$ & $40(16-88)$ & 0.62 \\
BUN (mg/dL) & $12.2(10.1-14.4)$ & $10.8(9.3-14.1)$ & 0.39 \\
SCr (mg/dL) & $0.77(0.61-0.86)$ & $0.65(0.57-0.76)$ & 0.11 \\
Na (mEq/L) & $141(139-143)$ & $141(139-144)$ & 0.52 \\
K (mEq/L) & $4.4(4.0-4.6)$ & $4.2(4.0-4.5)$ & 0.42 \\
Cl (mEq/L) & $104(101-106)$ & $106(104-107)$ & 0.02 \\
\hline
\end{tabular}

${ }^{\mathrm{a}}$ Data presented as median (range).

Table 4. Use of medication ${ }^{\mathrm{a}}$.

\begin{tabular}{|c|c|c|c|}
\hline Medications & $\begin{array}{l}\text { Low PN } \\
(N=24)\end{array}$ & $\begin{array}{c}\text { High PN } \\
(N=27)\end{array}$ & $P$ value \\
\hline Anti-hypertensive & $6(25.0)$ & $11(40.7)$ & 0.23 \\
\hline RA system inhibitors & $4(16.7)$ & $8(29.6)$ & 0.28 \\
\hline $\mathrm{Ca}$ antagonist & $4(16.7)$ & $6(22.2)$ & 0.44 \\
\hline Narcoleptic & $5(20.8)$ & $6(22.2)$ & 0.9 \\
\hline Anti-microbial & $5(20.8)$ & $4(14.8)$ & 0.42 \\
\hline Anti-diabetic & $0(0)$ & $2(7.4)$ & 0.28 \\
\hline Anti-hyperlipidemic & $1(4.2)$ & $0(0)$ & 0.47 \\
\hline Laxative & $7(29.2)$ & $9(33.3)$ & 0.75 \\
\hline Prokinetic & $4(16.7)$ & $9(33.3)$ & 0.17 \\
\hline NSAIDs & $9(37.5)$ & $5(18.5)$ & 0.13 \\
\hline Anti-diarrheal & $2(8.3)$ & $2(7.4)$ & 0.65 \\
\hline Anti-peptic ulcer & $9(37.5)$ & $8(29.6)$ & 0.55 \\
\hline Psychotropic & $4(16.7)$ & $2(7.4)$ & 0.28 \\
\hline Hematinic & $3(12.5)$ & $3(11.1)$ & 0.61 \\
\hline Narcotic & $2(8.3)$ & $0(0)$ & 0.22 \\
\hline Anti-flatulent & $3(12.5)$ & $3(11.1)$ & 0.61 \\
\hline
\end{tabular}

${ }^{\mathrm{a}}$ Data presented as $\mathrm{n}(\%)$.

\subsection{Factor Analysis for Predicting Peripheral Neuropathy}

To identify the predicting factors from observed variables, PCA was performed using the method of factor 
extraction with PASW software. The variables Na, ALT and BSA were excluded because they overlapped with $\mathrm{Cl}$, AST and oxaliplatin dose per course, respectively. Then, these variables were subjected to PCA. PCA revealed the presence of 15 components with eigenvalue exceeding 1 , explaining $81.1 \%$ of the variance. To simplify the item structure, varimax rotation was used following principal components factor extraction. Table 5 shows the rotated component matrix, representing the absolute value of factor loading in bold type if values were above 0.4 and in italic type if values were between 0.3 and 0.4 .

Each principal component and major variable is summarized in Table 6. For example, principal component 1 comprised variables $\gamma$-GTP (0.863), ALP (0.856), AST (0.836), LDH (0.762), cecum cancer type (0.585), antihyperlipidemic agent $(0.541)$ and psychotropic agent (0.470). Principal component 2 comprised the following variables: colon cancer type (0.964), rectum cancer type $(-0.877)$, and psychotropic agent (0.418). Principal component 3 comprised the following variables: laxative agent (0.808), antibacterial agent (0.655), narcoleptic agent $(-0.612)$, prokinetic agent $(0.611)$ and age $(0.424)$. Regarding peripheral neuropathy, principal component 9 yielded a loading value of -0.776 for high PN (Table 5 and Table 6). That is, it was suggested that variables such as $\mathrm{Cl}$ level and narcotic agent might be related to the incidence of peripheral neuropathy. Increase BMI, liver metastasis and lymph node metastasis were also related to the incidence of peripheral neuropathy because each loading value was above 0.3 . The positive factors for the incidence of peripheral neuropathy were serum $\mathrm{Cl}$ level, liver metastasis and lymph node metastasis. The use of a narcotic agent and BMI were negative factors.

\subsection{Discriminant Analysis of the Model Used to Predict Peripheral Neuropathy}

To build a predictive model for the group with a high frequency of peripheral neuropathy, the linear discriminant analysis was performed. Two models were generated for the integer-valued grouping variable of whether peripheral neuropathy appeared. Model equation A was based on the independent variable serum $\mathrm{Cl}$ level $(\mathrm{Cl}$ : $\mathrm{mEq} / \mathrm{L}$ ), and model equation $\mathrm{B}$ was derived from independent variables such as serum $\mathrm{Cl}$ level, usage of a narcotic agent (NA: yes $=1$, no $=0$ ), BMI $\left(\mathrm{kg} / \mathrm{m}^{2}\right)$, the status of liver metastasis $(\mathrm{LM}$ : yes $=1$, no $=0)$ and the status of lymph-node metastasis $(\mathrm{LNM}$ : yes $=1$, no $=0$ ). Each model was evaluated with cross-validation by the leave-one-out classification method.

Model equation A is:

$$
\mathrm{z}=(0.374 \times \mathrm{Cl})-39.075
$$

Model equation B is:

$$
\begin{aligned}
\mathrm{z}= & (0.211 \times \mathrm{Cl})+(1.310 \times \mathrm{LM})+(1.734 \times \mathrm{LNM}) \\
& +(-0.145 \times \mathrm{BMI})+(-1.090 \times \mathrm{NA})-19.608
\end{aligned}
$$

Model equation A had a $60.9 \%$ prediction rate in classifying the presence of peripheral neuropathy. Model equation B had a $76.4 \%$ prediction rate. After the crossvalidation, the rate of prediction success was $72.5 \%$.

\section{Discussion}

The primary purpose of this study was to investigate the predictive factors associated with the incidence of oxaliplatin-induced peripheral neuropathy, which is a major adverse event of oxaliplatin-base chemotherapy and is the most frequent dose-limiting toxicity in clinical practice. Peripheral sensory neuropathy is characterized by dysesthesia and/or distal paresthesia (fingers, toes and, less frequently, the peri-oral region and pharyngo-laryngeal tract), and induced or exacerbated cold. However, some patients treated with oxaliplatin show no or minimal peripheral neuropathy. These differences are poorly understood. In this study, 51 patients treated with the oxaliplatin-based regimen were retrospectively analyzed, and peripheral neuropathy was observed in 45 patients (88.2\%): 17 with grade 1 (33.1\%), 23 with grade 2 (45.1\%) and 5 with grade $3(9.8 \%)$. Six patients did not complain of peripheral neuropathy. According to the frequency of peripheral neuropathy, patients were divided to two groups: high-frequency and low-frequency peripheral neuropathy. In the high PN group, serum $\mathrm{Cl}$ level at baseline, and the total number of chemotherapy cycles were identified as predictive factors. However, the total number of chemotherapy cycles was not used because it was the variable used for assignment to the high $\mathrm{PN}$ and the low PN groups. Therefore, serum $\mathrm{Cl}$ level at baseline was identified as a predictive factor for the high PN group.

Oxaliplatin is normally administered by intravenous infusion, and it is transformed essentially by water and nucleophiles such as $\mathrm{Cl}^{-}$and $\mathrm{HCO}_{3}^{-}$[24]. Thus, oxaliplatin, in contrast to cisplatin, should not be mixed with solutions containing chloride. The proposed princepal mechanism of action of oxaliplatin is the formation of platinum-DNA adducts, resulting in cell death [25]. Oxaliplatin degradation in aqueous media depends on the chloride concentration and the $\mathrm{pH}[24,26]$. The major biotransformation products from oxaliplatin in the presence of chloride are [Pt(1,2-diaminocyclohexyl)Cl2] (dichloro complex) and oxalate [26]. The dichloro complex has been shown to be more cytotoxic than oxaliplatin in vitro [27]. Oxaliplatin altered voltage-gated $\mathrm{Na}^{+}$channel kinetics on rat sensory neurons [28]. Grolleau et al. showed that oxaliplatin is capable of altering the voltagegated $\mathrm{Na}^{+}$channels through a pathway involving calcium 
Table 5. Rotated component matrix.

\begin{tabular}{|c|c|c|c|c|c|c|c|c|c|c|c|c|c|c|c|}
\hline & \multicolumn{15}{|c|}{ Components $^{\mathrm{a}}$} \\
\hline & 1 & 2 & 3 & 4 & 5 & 6 & 7 & 8 & 9 & 10 & 11 & 12 & 13 & 14 & 15 \\
\hline$\gamma-\mathrm{GTP}$ & 0.863 & -0.061 & -0.051 & 0.036 & 0.198 & -0.026 & 0.171 & 0.173 & 0.064 & 0.053 & -0.075 & -0.011 & 0.068 & -0.062 & 0.012 \\
\hline ALP & 0.856 & 0.046 & -0.091 & -0.223 & 0.076 & -0.232 & 0 & -0.062 & -0.038 & -0.026 & -0.009 & -0.043 & -0.118 & -0.031 & -0.05 \\
\hline AST & 0.836 & 0.21 & 0.248 & 0.121 & -0.079 & 0.053 & -0.044 & -0.039 & 0.167 & 0.058 & -0.07 & -0.021 & -0.082 & 0.036 & -0.125 \\
\hline $\mathrm{LDH}$ & 0.762 & 0.117 & 0.174 & 0.099 & -0.209 & -0.012 & 0.021 & -0.063 & 0.02 & -0.011 & 0.038 & 0.17 & -0.098 & 0.057 & -0.27 \\
\hline Cecum cancer & 0.585 & -0.146 & -0.091 & -0.18 & 0.355 & 0.208 & 0.065 & 0.316 & -0.041 & 0.006 & -0.137 & -0.117 & 0.142 & -0.005 & 0.299 \\
\hline Narcotic & 0.525 & 0.306 & 0.216 & 0.179 & -0.206 & -0.076 & -0.104 & -0.246 & 0.405 & 0.058 & 0.016 & -0.072 & -0.05 & -0.021 & -0.32 \\
\hline Psychotropic & 0.47 & 0.418 & -0.096 & 0.272 & 0.056 & 0.103 & 0.22 & 0.086 & 0.299 & 0.054 & 0.31 & -0.096 & -0.13 & 0.259 & -0.129 \\
\hline Colon cancer & -0.014 & 0.964 & 0.048 & -0.06 & -0.001 & 0.026 & -0.038 & -0.017 & -0.028 & 0.032 & -0.024 & 0.004 & 0.013 & -0.006 & -0.061 \\
\hline Rectum cancer & -0.262 & -0.88 & -0.004 & 0.144 & -0.167 & -0.124 & 0.007 & -0.132 & 0.046 & -0.034 & 0.089 & 0.051 & -0.08 & 0.008 & -0.081 \\
\hline Laxative & 0.217 & 0.057 & 0.808 & 0.017 & 0.134 & 0.003 & 0.116 & 0.092 & -0.024 & 0.068 & 0.044 & -0.045 & 0.023 & -0.043 & -0.017 \\
\hline Antibacterial & -0.064 & 0.024 & 0.655 & 0.065 & 0.06 & -0.037 & -0.025 & -0.168 & 0.134 & -0.003 & 0.444 & -0.01 & 0.335 & -0.025 & 0.055 \\
\hline Narcoleptic & -0.119 & 0.011 & -0.61 & 0.165 & 0.209 & 0.008 & -0.116 & 0.103 & 0.043 & 0.368 & 0.143 & -0.266 & 0.038 & -0.077 & -0.069 \\
\hline Prokinetic & -0.251 & -0.074 & 0.611 & 0.128 & 0.148 & 0.153 & -0.065 & 0.008 & -0.13 & 0.25 & 0.079 & -0.217 & -0.099 & -0.283 & 0.08 \\
\hline Age & 0.145 & 0.227 & 0.424 & -0.051 & 0.368 & 0.141 & 0.156 & 0.369 & -0.288 & 0.243 & -0.08 & 0.172 & -0.182 & 0.099 & -0.194 \\
\hline Regimen 2 & -0.001 & 0.11 & -0.054 & -0.93 & -0.006 & 0.029 & 0.069 & -0.022 & 0.009 & 0.077 & -0.149 & -0.025 & -0.055 & -0.005 & -0.063 \\
\hline Regimen 1 & 0.047 & -0.031 & -0.019 & 0.84 & -0.04 & -0.097 & -0.035 & 0.06 & -0.001 & -0.107 & -0.47 & 0.004 & 0.05 & 0.052 & 0.026 \\
\hline Hematinic & -0.003 & -0.201 & -0.126 & 0.128 & -0.74 & 0.128 & 0.049 & 0.294 & -0.06 & -0.028 & -0.068 & 0.099 & -0.022 & -0.006 & 0.041 \\
\hline BUN & 0.141 & -0.017 & 0.095 & 0.111 & 0.653 & -0.031 & 0.152 & 0.202 & 0.07 & 0.15 & 0.082 & 0.27 & -0.087 & 0.167 & -0.063 \\
\hline Sex & -0.248 & 0.191 & -0.007 & -0.201 & -0.131 & 0.769 & 0.007 & -0.018 & 0.163 & -0.107 & 0.063 & -0.193 & 0.006 & -0.022 & 0.148 \\
\hline Oxaliplatin dose & -0.209 & -0.031 & -0.053 & -0.118 & -0.128 & -0.67 & -0.245 & -0.056 & 0.286 & 0.065 & -0.15 & 0.119 & 0.216 & -0.009 & 0.137 \\
\hline Anti-peptic ulcer & 0 & -0.155 & 0.043 & 0.025 & -0.31 & 0.562 & -0.264 & 0.095 & 0.207 & -0.055 & 0.055 & 0.288 & 0.065 & 0.017 & 0.046 \\
\hline $\mathrm{Cl}$ & -0.203 & 0.204 & 0.047 & -0.047 & 0.101 & 0.529 & -0.092 & 0.065 & -0.44 & 0.117 & -0.083 & -0.06 & 0.361 & -0.111 & 0.074 \\
\hline T.Bil. & 0.111 & 0.015 & 0.133 & -0.084 & -0.072 & -0.067 & 0.858 & 0.13 & 0.2 & 0.071 & 0.018 & -0.065 & -0.035 & 0.069 & 0.119 \\
\hline Lung meta & -0.044 & -0.064 & 0.086 & -0.15 & 0.299 & 0.085 & 0.636 & -0.291 & -0.05 & 0.196 & -0.108 & 0.231 & -0.31 & -0.04 & 0.113 \\
\hline Anti-hyperlipidemic & 0.541 & -0.093 & -0.025 & 0.069 & 0.278 & 0.076 & 0.564 & 0.362 & -0.018 & -0.085 & 0.032 & 0.002 & 0.103 & -0.004 & 0.111 \\
\hline $\mathrm{Ca}$ antagonist & 0.052 & 0.057 & 0.007 & 0.094 & -0.082 & 0.088 & 0.011 & 0.876 & 0.007 & 0.043 & -0.11 & -0.08 & -0.195 & -0.097 & 0.095 \\
\hline High PN & -0.131 & 0.048 & 0.065 & 0.025 & -0.075 & -0.035 & -0.123 & 0.027 & -0.78 & 0.087 & 0 & -0.185 & -0.042 & 0.074 & -0.02 \\
\hline BMI & 0.012 & 0.206 & 0.108 & -0.37 & 0.145 & -0.104 & 0.199 & 0.267 & 0.391 & 0.239 & 0.309 & -0.073 & 0.135 & 0.054 & 0.245 \\
\hline Anti-diarrheal & -0.112 & -0.162 & -0.145 & 0.091 & -0.081 & -0.016 & -0.047 & -0.014 & 0.064 & -0.85 & -0.025 & -0.081 & -0.118 & -0.053 & 0.128 \\
\hline Anti-flatulent & 0.047 & 0.113 & 0.096 & 0.148 & -0.024 & 0.174 & -0.12 & -0.04 & 0.027 & -0.75 & -0.082 & -0.063 & -0.083 & -0.038 & -0.38 \\
\hline Regimen 3 & -0.071 & -0.094 & 0.097 & -0.095 & 0.068 & 0.111 & -0.035 & -0.064 & -0.009 & 0.065 & 0.908 & 0.025 & -0.007 & -0.074 & 0.041 \\
\hline Past RadioT & -0.067 & -0.073 & -0.093 & 0.075 & 0.108 & 0.026 & -0.025 & -0.065 & 0.17 & 0.101 & -0.119 & 0.77 & -0.034 & -0.093 & 0.008 \\
\hline Past ChemoT & 0.129 & 0.117 & 0.202 & -0.303 & -0.32 & -0.216 & -0.045 & -0.026 & 0.021 & 0.057 & 0.279 & 0.61 & 0.183 & 0.051 & 0.071 \\
\hline $\mathrm{SCr}$ & -0.03 & -0.047 & -0.021 & 0.187 & 0.376 & -0.34 & 0.219 & 0.032 & -0.008 & -0.084 & 0.216 & 0.535 & 0.184 & 0.193 & 0.07 \\
\hline NSAIDs & -0.008 & -0.073 & 0.144 & -0.031 & 0.132 & 0.048 & -0.17 & -0.179 & 0.259 & 0.106 & -0.028 & 0.221 & 0.737 & -0.017 & -0.016 \\
\hline
\end{tabular}




\begin{tabular}{|c|c|c|c|c|c|c|c|c|c|c|c|c|c|c|c|}
\hline \multicolumn{16}{|l|}{ Continued } \\
\hline Lymph node Meta & -0.106 & 0.16 & -0.034 & 0.124 & -0.237 & -0.088 & 0.008 & -0.019 & -0.32 & 0.111 & 0.102 & -0.101 & 0.652 & -0.109 & 0.098 \\
\hline Liver Meta & 0.393 & -0.06 & 0.201 & -0.115 & 0.031 & -0.007 & -0.31 & 0.161 & -0.33 & -0.261 & 0.189 & -0.005 & -0.42 & 0.141 & 0.139 \\
\hline Anti-diabetic & -0.137 & 0.124 & -0.139 & 0.156 & 0.109 & -0.137 & 0.093 & -0.084 & -0.103 & 0.143 & 0.017 & -0.079 & -0.112 & 0.767 & 0.044 \\
\hline K & 0.179 & -0.35 & 0.05 & -0.101 & 0.071 & 0.226 & -0.159 & -0.027 & 0.113 & -0.094 & -0.248 & 0.064 & -0.057 & 0.681 & 0.022 \\
\hline RA system Inh. & 0.021 & 0.211 & -0.056 & -0.199 & -0.039 & -0.168 & 0.201 & 0.516 & -0.207 & 0.015 & 0.15 & 0.042 & 0.107 & 0.516 & -0.283 \\
\hline Past Ope. & -0.22 & 0.017 & 0.081 & 0.097 & -0.098 & 0.068 & 0.161 & 0.034 & 0.009 & 0.072 & 0.056 & 0.064 & 0.019 & 0 & 0.87 \\
\hline Eigenvalues & 5.59 & 3.61 & 3.04 & 2.94 & 2.47 & 2.31 & 2.02 & 1.79 & 1.72 & 1.65 & 1.53 & 1.28 & 1.17 & 1.09 & 1.02 \\
\hline $\begin{array}{c}\text { Contribution ratio } \\
(\%)\end{array}$ & 13.63 & 8.82 & 7.42 & 7.18 & 6.03 & 5.64 & 4.93 & 4.36 & 4.2 & 4.01 & 3.73 & 3.13 & 2.86 & 2.67 & 2.5 \\
\hline CCR (\%) & 13.63 & 22.45 & 29.87 & 37.05 & 43.08 & 48.72 & 53.65 & 58.01 & 62.21 & 66.22 & 69.96 & 73.09 & 75.95 & 78.62 & 81.11 \\
\hline
\end{tabular}

${ }^{\mathrm{a}}$ The absolute value of factor loading in bold type if values were above 0.4 and in italic type if values were between 0.3 and 0.4 ; ${ }^{\mathrm{b}}$ Cumulative contribution ratio.

Table 6. Principal components and major variables.

\begin{tabular}{|c|c|}
\hline $\begin{array}{l}\text { Principal } \\
\text { Components }\end{array}$ & Variables $^{\mathrm{a}}$ \\
\hline 1 & $\begin{array}{l}\gamma \text {-GTP, ALP, AST, LDH, Cecum cancer, Narcotic, } \\
\text { Psychotropic, Anti-hyperlipidemic, Liver meta }\end{array}$ \\
\hline 2 & $\begin{array}{l}\text { Colon cancer, Rectum cancer, Psychotropic, } \underline{K} \text {, } \\
\text { Narcotic }\end{array}$ \\
\hline 3 & Laxative, Anti-bacterial, Narcoleptic, Prokinetic, Age \\
\hline 4 & 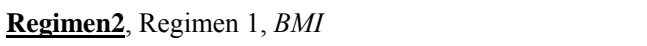 \\
\hline 5 & $\begin{array}{l}\text { Hematinic, BUN, SCr, Age, Cecum cancer, } \underline{\text { Past }} \\
\text { Chemotherapy, } \underline{\text { Anti-peptic ulcer }}\end{array}$ \\
\hline 6 & Sex, Oxaliplatin dose, Anti-peptic ulcer, $\mathrm{Cl}, \underline{S C r}$ \\
\hline 7 & T.Bil, Lung meta, Anti-hyperlipidemic, $\underline{\text { Liver meta }}$ \\
\hline 8 & Ca antagonist, Age, Anti-hyperlipidemic, Cecum cancer \\
\hline 9 & $\underline{\underline{\text { High PN }}}, \underline{\text { Cl}}$, Narcotic, $B M I, \underline{\text { Liver meta }}, \underline{\text { Lymph node }}$ \\
\hline 10 & Anti-diarrheal, Anti-flatulent, Narcoleptic \\
\hline 11 & $\begin{array}{l}\text { Regimen3, Regimen1, Antibacterial, Psychotropic, } \\
B M I\end{array}$ \\
\hline 12 & Past radiotherapy, Past chemotherapy, SCr \\
\hline 13 & $\begin{array}{l}\text { NSAIDs, Lymph node meta, Liver meta, } \mathrm{Cl} \text {, } \\
\text { Antibacterial, } \underline{\text { Lung meta }}\end{array}$ \\
\hline 14 & Anti-diabetic, K, RA system inhibitor \\
\hline 15 & Past operation, Anti-flatulent, Narcotic \\
\hline
\end{tabular}

${ }^{a}$ The absolute value of factor loading in bold type if values were above 0.4 and in italic type if values were between 0.3 and 0.4 in Table 5. Underlined variables show negative factors as shown in Table 5.

ions, which are probably immobilized by the oxalate [29]. Recently, Sakurai et al. showed that oxalate is involved in oxaliplatin-induced cold hyperalgesia/allodynia but not mechanical allodynia through the chelation of $\mathrm{Ca}^{2+}$ and $\mathrm{Mg}^{2+}$. However, the dichloro complex induces me- chanical allodynia but not cold hyperalgesia/allodynia [30]. The degradation of oxaliplatin might be affected by the high serum $\mathrm{Cl}$ level, and the resulting oxalate might be involved in the incidence of peripheral neuropathy. Therefore, monitoring the serum $\mathrm{Cl}$ level in patients $(>105 \mathrm{mEq} / \mathrm{L}$ from model equation $\mathrm{A}$ ) might be useful in predicting peripheral neuropathy.

\section{Conclusions}

Our retrospective analysis indicates that the serum $\mathrm{Cl}$ level at baseline, use of a narcotic agent, BMI, status of liver metastasis, and status of lymph node metastasis are predictive factors for peripheral neuropathy associated with the administration of oxaliplatin. Our predictive model equation achieved $60.9 \%$ and $76.4 \%$ prediction rates. This equation may be useful for predicting the incidence of peripheral neuropathy in clinical practice. Understanding the patient's characteristics could be useful in predicting and preventing oxaliplatin-induced peripheral neuropathy.

\section{Ethics Approval}

The study design was reviewed by a local ethics committee for research involving human subjects, Graduate School of Pharmaceutical Sciences, Tohoku University and South Miyagi Medical Center.

\section{REFERENCES}

[1] C. Tournigand, T. Andre, E. Achille, G. Lledo, M. Flesh, D. Mery-Mignard, E. Quinaux, C. Couteau, M. Buyse, G. Ganem, B. Landi, P. Colin, C. Louvet and A. de Gramont, "FOLFIRI Followed by FOLFOX6 or the Reverse Sequence in Advanced Colorectal Cancer: A Randomized GERCOR Study," Journal of Clinical Oncology, Vol. 22, No. 2, 2004, pp. 229-237. doi: 10.1200/JCO.2004.05.113

[2] A. de Gramont, A. Figer, M. Seymour, M. Homerin, A. 
Hmissi, J. Cassidy, C. Boni, H. Cortes-Funes, A. Cervantes, G. Freyer, D. Papamichael, N. Le Bail, C. Louvet, D. Hendler, F. de Braud, C. Wilson, F. Morvan and A. Bonetti, "Leucovorin and Fluorouracil with or without Oxaliplatin as First-Line Treatment in Advanced Colorectal Cancer," Journal of Clinical Oncology, Vol. 18, No. 16, 2000, pp. 2938-2947.

[3] R. M. Goldberg, D. J. Sargent, R. F. Morton, C. S. Fuchs, R. K. Ramanathan, S. K. Williamson, B. P. Findlay, H. C. Pitot and S. R. Alberts, "A Randomized Controlled Trial of Fluorouracil plus Leucovorin, Irinotecan, and Oxaliplatin Combinations in Patients with Previously Untreated Metastatic Colorectal Cancer," Journal of Clinical Oncology, Vol. 22, No. 1, 2004, pp. 23-30. doi: 10.1200/JCO.2004.09.046

[4] L. B. Saltz, S. Clarke, E. Diaz-Rubio, W. Scheithauer, A. Figer, R. Wong, S. Koski, M. Lichinitser, T. S. Yang, F. Rivera, F. Couture, F. Sirzen and J. Cassidy, "Bevacizumab in Combination with Oxaliplatin-Based Chemotherapy as First-Line Therapy in Metastatic Colorectal Cancer: A Randomized Phase III Study," Journal of Clinical Oncology, Vol. 26, No. 12, 2008, pp. 2013-2019. doi: 10.1200/JCO.2007.14.9930

[5] J. Cassidy, S. Clarke, E. Diaz-Rubio, W. Scheithauer, A. Figer, R. Wong, S. Koski, M. Lichinitser, T. S. Yang, F. Rivera, F. Couture, F. Sirzen and L. Saltz, "Randomized Phase III Study of Capecitabine plus Oxaliplatin Compared with Fluorouracil/Folinic Acid plus Oxaliplatin as First-Line Therapy for Metastatic Colorectal Cancer," Journal of Clinical Oncology, Vol. 26, No. 12, 2008, pp. 2006-2012. doi: 10.1200/JCO.2007.14.9898

[6] Y. Kidani, M. Noji and T. Tashiro, "Antitumor Activity of Platinum(II) Complexes of 1,2-Diamino-cyclohexane Isomers," Gann, Vol. 71, No. 5, 1980, pp. 637-643.

[7] A. Ibrahim, S. Hirschfeld, M. H. Cohen, D. J. Griebel, G. A. Williams and R. Pazdur, "FDA Drug Approval Summaries: Oxaliplatin," Oncologist, Vol. 9, No. 1, 2004, pp. 8-12. doi: 10.1634/theoncologist.9-1-8

[8] L. Gamelin, M. Boisdron-Celle, A. Morel and E. Gamelin, "Oxaliplatin Neurotoxicity," Bull Cancer, Vol. 93, Suppl. 1, 2006, pp. S17-22.

[9] L. Gamelin, M. Boisdron-Celle, R. Delva, V. GuerinMeyer, N. Ifrah, A. Morel and E. Gamelin, "Prevention of Oxaliplatin-Related Neurotoxicity by Calcium and Magnesium Infusions: A Retrospective Study of 161 Patients Receiving Oxaliplatin Combined with 5-Fluorouracil and Leucovorin for Advanced Colorectal Cancer," Clinical Cancer Research, Vol. 10, No. 12, Part 1, 2004, pp. 4055-4061.

[10] H. S. Hochster, A. Grothey and B. H. Childs, "Use of Calcium and Magnesium Salts to Reduce OxaliplatinRelated Neurotoxicity," Journal of Clinical Oncology, Vol. 25, No. 25, 2007, pp. 4028-4029. doi: 10.1200/JCO.2007.13.5251

[11] C. Gedlicka, W. Scheithauer, B. Schull and G. V. Kornek, "Effective Treatment of Oxaliplatin-Induced Cumulative Polyneuropathy with Alpha-Lipoic Acid," Journal of Clinical Oncology, Vol. 20, No. 15, 2002, pp. 3359-3361. doi: $10.1200 /$ JCO.2002.99.502
[12] J. P. Durand, C. Brezault and F. Goldwasser, "Protection against Oxaliplatin Acute Neurosensory Toxicity by Venlafaxine," Anticancer Drugs, Vol. 14, No. 6, 2003, pp. 423-425. doi: 10.1097/00001813-200307000-00006

[13] J. P. Durand, G. Deplanque, V. Montheil, J. M. Gornet, F. Scotte, O. Mir, A. Cessot, R. Coriat, E. Raymond, E. Mitry, P. Herait, Y. Yataghene and F. Goldwasser, "Efficacy of Venlafaxine for the Prevention and Relief of Oxaliplatin-Induced Acute Neurotoxicity: Results of EFFOX, a Randomized, Double-Blind, Placebo-Controlled Phase III Trial," Annals of Oncology, 2011.

[14] M. Penz, G. V. Kornek, M. Raderer, H. Ulrich-Pur, W. Fiebiger and W. Scheithauer, "Subcutaneous Administration of Amifostine: A Promising Therapeutic Option in Patients with Oxaliplatin-Related Peripheral Sensitive Neuropathy," Annals of Oncology, Vol. 12, No. 3, 2001, pp. 421-422. doi: 10.1023/A:1011184609963

[15] S. Cascinu, F. Graziano, F. Ferrau, V. Catalano, C. Massacesi, D. Santini, R. R. Silva, S. Barni, A. Zaniboni, N. Battelli, S. Siena, P. Giordani, D. Mari, A. M. Baldelli, S. Antognoli, R. Maisano, D. Priolo, M. A. Pessi, G. Tonini, S. Rota and R. Labianca, "Raltitrexed plus Oxaliplatin (TOMOX) as First-Line Chemotherapy for Metastatic Colorectal Cancer. A Phase II Study of the Italian Group for the Study of Gastrointestinal Tract Carcinomas (GISCAD)," Annals of Oncology, Vol. 13, No. 5, 2002, pp. 716-720. doi: 10.1093/annonc/mdf091

[16] W. S. Wang, J. K. Lin, T. C. Lin, W. S. Chen, J. K. Jiang, H. S. Wang, T. J. Chiou, J. H. Liu, C. C. Yen and P. M. Chen, "Oral Glutamine Is Effective for Preventing Oxaliplatin-Induced Neuropathy in Colorectal Cancer Patients," Oncologist, Vol. 12, No. 3, 2007, pp. 312-319. doi: 10.1634/theoncologist.12-3-312

[17] S. von Delius, F. Eckel, S. Wagenpfeil, M. Mayr, K. Stock, F. Kullmann, F. Obermeier, J. Erdmann, R. Schmelz, S. Quasthoff, H. Adelsberger, R. Bredenkamp, R. M. Schmid and C. Lersch, "Carbamazepine for Prevention of Oxaliplatin-Related Neurotoxicity in Patients with Advanced Colorectal Cancer: Final Results of a Randomised, Controlled, Multicenter Phase II Study," Invest New Drugs, Vol. 25, No. 2, 2007, pp. 173-180. doi: 10.1007/s10637-006-9010-y

[18] P. L. Mitchell, D. Goldstein, M. Michael, P. Beale, M. Friedlander, J. Zalcberg, S. White, J. A. Thomson and S. Clarke, "Addition of Gabapentin to a Modified FOLFOX Regimen Does Not Reduce Oxaliplatin-Induced Neurotoxicity," Clinical Colorectal Cancer, Vol. 6, No. 2, 2006, pp. 146-151. doi: 10.3816/CCC.2006.n.032

[19] M. W. Saif and S. Hashmi, "Successful Amelioration of Oxaliplatin-Induced Hyperexcitability Syndrome with the Antiepileptic Pregabalin in a Patient with Pancreatic Cancer," Cancer Chemotherapy and Pharmacology, Vol. 61, No. 3, 2008, pp. 349-354. doi: 10.1007/s00280-007-0584-7

[20] M. W. Saif, K. Syrigos, K. Kaley and I. Isufi, "Role of Pregabalin in Treatment of Oxaliplatin-Induced Sensory Neuropathy," Anticancer Research, Vol. 30, No. 7, 2010, pp. 2927-2933.

[21] C. Tournigand, A. Cervantes, A. Figer, G. Lledo, M. 
Flesch, M. Buyse, L. Mineur, E. Carola, P. L. Etienne, F. Rivera, I. Chirivella, N. Perez-Staub, C. Louvet, T. Andre, I. Tabah-Fisch and A. de Gramont, "OPTIMOX1: A Randomized Study of FOLFOX4 or FOLFOX7 with Oxaliplatin in a Stop-and-Go Fashion in Advanced Colorectal Cancer-A GERCOR Study," Journal of Clinical Oncology, Vol. 24, No. 3, 2006, pp. 394-400. doi: 10.1200/JCO.2005.03.0106

[22] A. De Gramont, M. Krulik, J. Cady, B. Lagadec, J. E. Maisani, J. P. Loiseau, J. D. Grange, G. Gonzalez-Canali, B. Demuynck, C. Louvet, et al., "High-Dose Folinic Acid and 5-Fluorouracil Bolus and Continuous Infusion in Advanced Colorectal Cancer," European Journal of Cancer and Clinical Oncology, Vol. 24, No. 9, 1988, pp. 1499-1503. doi: 10.1016/0277-5379(88)90341-0

[23] T. Shimizu, T. Satoh, K. Tamura, T. Ozaki, I. Okamoto, M. Fukuoka and K. Nakagawa, "Oxaliplatin/Fluorouracil/Leucovorin (FOLFOX4 and Modified FOLFOX6) in Patients with Refractory or Advanced Colorectal Cancer: Post-Approval Japanese Population Experience," International Journal of Clinical Oncology, Vol. 12, No. 3, 2007, pp. 218-223. doi: 10.1007/s10147-007-0658-X

[24] E. Jerremalm, M. Hedeland, I. Wallin, U. Bondesson and H. Ehrsson, "Oxaliplatin Degradation in the Presence of Chloride: Identification and Cytotoxicity of the Monochloro Monooxalato Complex," Pharmaceutical Research, Vol. 21, No. 5, 2004, pp. 891-894. doi: 10.1023/B:PHAM.0000026444.67883.83

[25] S. Mani, M. A. Graham, D. B. Bregman, P. Ivy and S. G.
Chaney, "Oxaliplatin: A Review of Evolving Concepts," Cancer Invest, Vol. 20, No. 2, 2002, pp. 246-263. doi: 10.1081/CNV-120001152

[26] M. E. Alberto, M. F. Lucas, M. Pavelka and N. Russo, "The Degradation Pathways in Chloride Medium of the Third Generation Anticancer Drug Oxaliplatin," Journal of Physical Chemistry B, Vol. 112, No. 35, 2008, pp. 10765-10768. doi: 10.1021/jp800476b

[27] F. R. Luo, S. D. Wyrick and S. G. Chaney, "Cytotoxicity, Cellular Uptake, and Cellular Biotransformations of Oxaliplatin in Human Colon Carcinoma Cells," Oncology Research, Vol. 10, No. 11-12, 1998, pp. 595-603.

[28] H. Adelsberger, S. Quasthoff, J. Grosskreutz, A. Lepier, F. Eckel and C. Lersch, "The Chemotherapeutic Oxaliplatin Alters Voltage-Gated $\mathrm{Na}(+)$ Channel Kinetics on Rat Sensory Neurons," European Journal of Pharmacology, Vol. 406, No. 1, 2000, pp. 25-32. doi: 10.1016/S0014-2999(00)00667-1

[29] F. Grolleau, L. Gamelin, M. Boisdron-Celle, B. Lapied, M. Pelhate and E. Gamelin, "A Possible Explanation for a Neurotoxic Effect of the Anticancer Agent Oxaliplatin on Neuronal Voltage-Gated Sodium Channels," Journal of Neurophysiology, Vol. 85, No. 5, 2001, pp. 2293-2297.

[30] M. Sakurai, N. Egashira, T. Kawashiri, T. Yano, H. Ikesue and R. Oishi, "Oxaliplatin-Induced Neuropathy in the Rat: Involvement of Oxalate in Cold Hyperalgesia But Not Mechanical Allodynia," Pain, Vol. 147, No. 1-3, 2009, pp. 165-174. doi: 10.1016/j.pain.2009.09.003 